\title{
Expression levels of Activated Leukocyte Cell Adhesion Molecule (ALCAM/CD166) in primary breast carcinoma and distant breast
} cancer metastases

\author{
M. Ihnen ${ }^{\mathrm{a}, *}$, N. Köhler ${ }^{\mathrm{a}}$, J.F. Kersten ${ }^{\mathrm{c}}$, K. Milde-Langosch ${ }^{\mathrm{a}}$, K. Beck ${ }^{\mathrm{a}}$, S. Höller ${ }^{\mathrm{b}}$, V. Müller ${ }^{\mathrm{a}}$, \\ I. Witzel ${ }^{\mathrm{a}}$, F. Jänicke ${ }^{\mathrm{a}}$ and E. Kilic ${ }^{\mathrm{b}}$ \\ ${ }^{a}$ Department of Gynecology, University Medical Center Hamburg-Eppendorf, Martinistr. 52, D-20246 Hamburg, \\ Germany \\ b Institute for Pathology, University Hospital of Basel, Schönbeinstrasse 40, 4031 Basel, Switzerland \\ ${ }^{c}$ Department of Statistics and Epidemiology, University Medical Center Hamburg-Eppendorf, Martinistr. 52, \\ D-20246 Hamburg, Germany
}

\begin{abstract}
Introduction: Activated Leukocyte Cell Adhesion Molecule (ALCAM/CD166) gained increasing attention regarding tumorprogression and metastatic spread in breast cancer. The aim of this study was to examine ALCAM expression levels in primary breast cancer and distant metastases of the same patient within 29 autopsy cases to better understand the underlying mechanisms of metastases and the role of adhesion molecules in this process.

Material and Methods: Paraffin-embedded tissue of the primary and distant metastases $(\mathrm{N}=84)$ were collected and ALCAM immunohistochemistry was performed.

Results: The primary tumor and all metastases showed a statistically normally distributed ALCAM expression. ALCAM expression level average differs between immunoreactive score (IRS) (mean) 4.16 (lung)-5.00 (adrenal gland). Of the metastatic ALCAM expression levels we obtained an intra-class correlation (ICC) of $80.9 \%$, indicating a strong cluster effect of measurements in the same patient. ALCAM expression scores in metastatic sites and in the primary analyzed by hierarchical regression analysis showed that ALCAM expression in the primary is prognostic for ALCAM expression in all different sites of metastases (slope = $\left.0.773, p<0.001, r^{2}=0.504\right)$.

Conclusion: ALCAM expression in the primary is positively correlated to ALCAM expression in metastases within one single patient. This could show a tumorbiological context of ALCAM for the development of metastases in breast cancer.
\end{abstract}

Keywords: Adhesion molecules, ALCAM, breast cancer, distant metastasis, metastasis development

\section{Introduction}

Adhesion molecules like the Activated Leukocyte Cell Adhesion Molecule (ALCAM) are important for cell growth, cell survival and motility, and also for in-

* Corresponding author: M. Ihnen MD, Department of Gynecology, University Medical Center Hamburg-Eppendorf, Martinistr. 52, D-20246 Hamburg, Germany. Tel.: +49 1799153286; Fax: +49 40 7410 54355; E-mail: m.ihnen@uke.uni-hamburg.de. vasion during tumor progression and metastases [1]. Loss, overexpression, or malfunction of adhesion molecules may contribute to the detachment of tumor cells and therefore to local invasion and tumorprogression [2]. It was observed that antibodies against adhesion molecules like ALCAM decreased proliferation of breast cancer cells but also decreased the adhesion of breast cancer cells to each other [3]. ALCAM, a glycoprotein of the immunoglobulin superfamily of adhesion molecules, is mapped to human chromosome $3 q 13$, has five extracellular immunoglobulin do- 


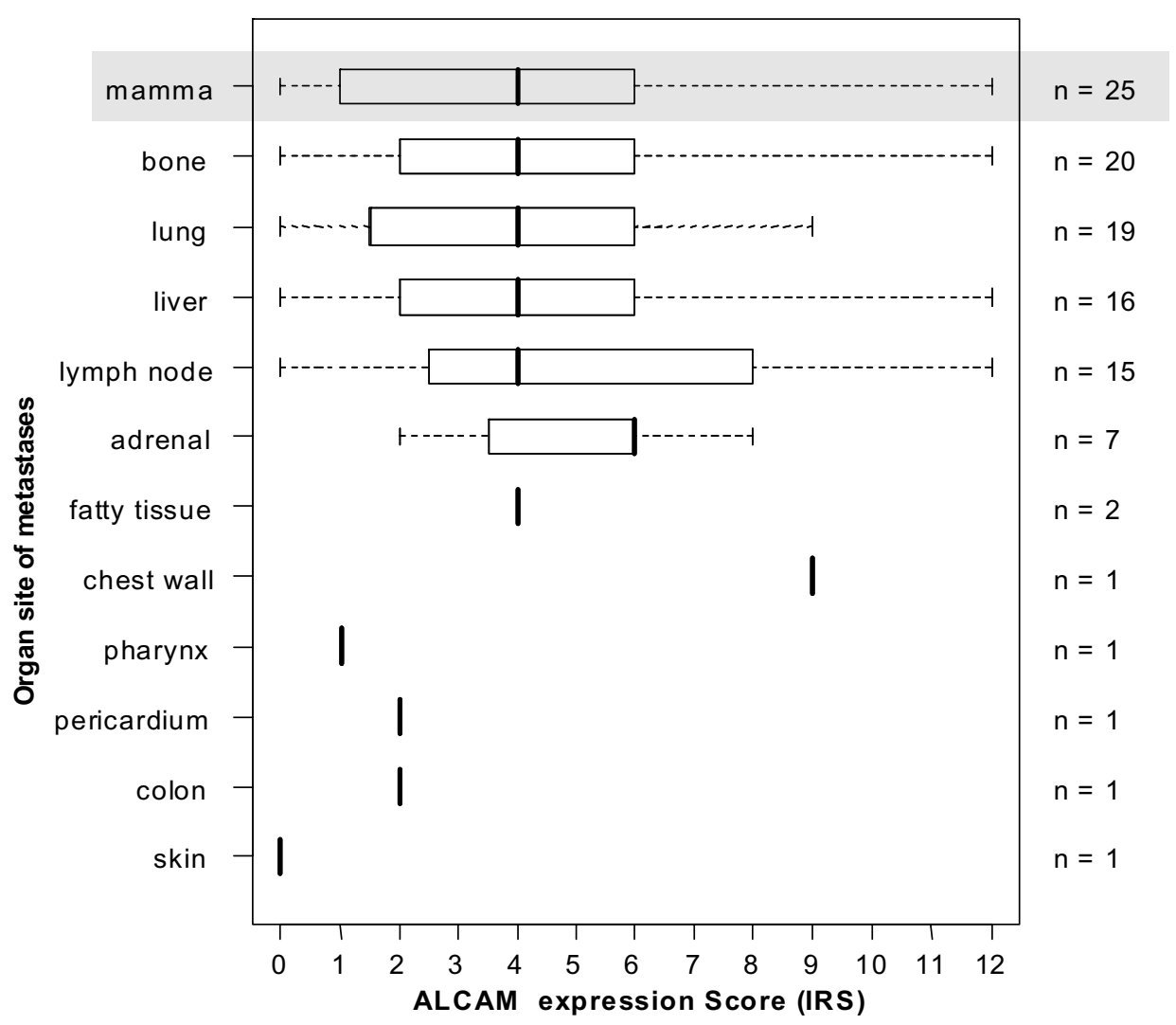

Fig. 1. Boxplot analysis of ALCAM expression levels (IRS) in different sites of metastases and the primary (mamma tissue). Median, standard deviation, minimum and maximum are shown.

mains (two $\mathrm{NH}_{2}$-terminal, membrane-distal variable[V]-type folds and three membrane-proximal constant[C2]-type immunoglobulin folds), a transmembrane region, and a short cytoplasmic tail $[4,5]$. It is involved in both homotypic/homophilic (to ALCAM) and heterotypic/heterophilic (to CD6) adhesions. Its physiological expression has been described in subsets of cells being involved in dynamic growth and migration [6$10]$.

In neoplasms like malignant melanoma [11], prostate cancer [12], colorectal carcinoma [13], esophageal squamous cell carcinoma [14] and only very recently in breast carcinoma a pathologically altered ALCAM expression has been observed and could be associated with tumorgenesis and tumorprogession [12-15]. So far ALCAM expression in neoplasms like breast cancer has always been analysed in the primary tumor but never in distant metastases within one single individual nor in distant metastases of the same site. This is content of this work because ALCAM may have an influence on metastasic site and metastasic pattern due to its appending properties that depend on the ALCAM expression in situ and might therefore be a possible target for further therapy options, which recently could be shown for another adhesion molecule namely Carcinoembryonic antigen-related cell adhesion molecule 6 (CEACAM6) in pancreatic cancer [16].

In this study we focused on ALCAM expression in the primary breast tumor with respect to the corresponding ALCAM expression in various distant metastases within the same individual.

\section{Material and methods}

\subsection{Patients}

Formalin-fixed, paraffin-embedded (FFPE) tissue of primary breast cancer $(\mathrm{N}=25)$ and distant breast cancer metastases $(\mathrm{N}=84)$ (at average $\sim 3$ metastasis per patient) were available from 29 autopsy cases, see Table 1, Fig. 1. The autopsies were performed between 1997 and 2005 at the Department of Pathology, University Hospital Basel. Advanced breast cancer was the 
Table 1

Distribution of ALCAM expression scores (IRS) in disseminated metastases from different organ sites and the primary breast carcinoma of 29 patients

\begin{tabular}{|c|c|c|c|c|c|c|c|c|c|c|c|c|}
\hline $\begin{array}{c}\text { Organ } \\
\text { Patient } \\
\text { No. }\end{array}$ & $\begin{array}{c}\text { Mamma } \\
\text { (Primary) } \\
\mathrm{N}=25\end{array}$ & $\begin{array}{c}\text { Bone } \\
\mathrm{N}=20\end{array}$ & $\begin{array}{c}\text { Lung } \\
\mathrm{N}=19\end{array}$ & $\begin{array}{c}\text { Liver } \\
\mathrm{N}=16\end{array}$ & $\begin{array}{l}\text { Lymph } \\
\text { node } \\
\mathrm{N}=20\end{array}$ & $\begin{array}{c}\text { Adrenal } \\
\mathrm{N}=7\end{array}$ & $\begin{array}{l}\text { Skin } \\
N=1\end{array}$ & $\begin{array}{l}\text { Colon } \\
\mathrm{N}=2\end{array}$ & $\begin{array}{l}\text { Pericardium } \\
\mathrm{N}=1\end{array}$ & $\begin{array}{c}\text { Pharynx } \\
\mathrm{N}=1\end{array}$ & $\begin{array}{c}\text { Chest wall } \\
\mathrm{N}=1\end{array}$ & $\begin{array}{l}\text { Fatty } \\
\text { tissue } \\
\mathrm{N}=2\end{array}$ \\
\hline 1 & 6 & 3 & 9 & 6 & & 4 & & & & & & \\
\hline 2 & & & 1 & 2 & 3 & & & & & & & \\
\hline 3 & 6 & & 6 & & 4 & & & & & & & \\
\hline 4 & & 6 & & 6 & 2 & & & & & & & \\
\hline 5 & 4 & 1 & & & & & & & & & & \\
\hline 6 & 12 & 12 & & 12 & 12 & & & & & & & \\
\hline 7 & 1 & & 4 & 1 & & & & & & & & \\
\hline 8 & 9 & 12 & & & 9 & 8 & & & & & & \\
\hline 9 & 4 & 2 & 2 & 4 & 1 & & & & & & & \\
\hline 10 & 1 & 1 & 3 & 1 & 3 & & & & & & & \\
\hline 11 & 6 & 0 & 0 & 0 & & & & & & & & \\
\hline 12 & 6 & 9 & & 6 & & & & & & & & \\
\hline 13 & 2 & 1 & 0 & & & & & & & & & \\
\hline 14 & 4 & 6 & & 4 & & & & & & & & \\
\hline 15 & & & 9 & & 8 & & & & & & 9 & \\
\hline 16 & 8 & & 9 & 6 & & 6 & & & & & & \\
\hline 17 & 4 & 3 & 2 & 3 & & 3 & & & & & & 4 \\
\hline 18 & & 3 & 3 & & & 3 & & 2 & & & & \\
\hline 19 & 4 & 6 & 0 & & 4 & & & & & & & \\
\hline 20 & 4 & 4 & 6 & & & 6 & & & & & & \\
\hline 21 & 3 & & & & 8 & 6 & & & & & & \\
\hline 22 & 1 & & 1 & & & & & & & 1 & & \\
\hline 23 & 1 & & 2 & & & 2 & & 2 & 2 & & & \\
\hline 24 & 1 & 2 & & & 2 & & & & & & & \\
\hline 25 & 6 & 6 & 6 & 6 & & & & & & & & 6 \\
\hline 26 & 8 & 4 & & & 4 & & & & & & & \\
\hline 27 & 1 & 4 & 6 & & & & & & & & & \\
\hline 28 & 0 & & 0 & & 0 & & 0 & & & & & \\
\hline 29 & 6 & 9 & 9 & 9 & 9 & & & & & & & \\
\hline
\end{tabular}

cause of death of all patients in this study. In $17 \%$ (5 cases) of all cases the initial diagnosis of breast cancer was made during autopsy. In all other cases the primary was excised during the initial diagnosis before the patients died of breast cancer and got post-mortem examination. In $14 \%$ (4 cases) of all cases there was no primary breast carcinoma tissue available but metastases were obtained from different sites. The Median age of all patients was 65 years at time of death (43-93 years). All primary tumors were poorly differentiated (grade 3 according to Elston and Ellis [22]) invasive ductal carcinomas.

The study was performed in accordance with the principles of the declaration of Helsinki after approval by the local ethics committee.

\subsection{Immunhistochemistry}

Formalin fixed, paraffin embedded tissue (FFPE) sections of primary breast cancer tumors and breast cancer metastases were cut at a thickness of $5 \mu \mathrm{m}$ and mounted on slides (Superfrost/Plus, Sondheim Ger- many), dewaxed with Xylene and gradually hydrated. Slides were then placed in boiling $10 \mathrm{mM}$ citrate buffer for thirty minutes, washed in Aqua dest. and then placed in $0.5 \% \mathrm{H}_{2} \mathrm{O}_{2}$-Methanol solution for $30 \mathrm{~min}$ utes and washed again in TRIS buffered saline (TBS). Then a blocking solution including TBS and Normal Serum (Vectastatin Elite ABC mouse Kit) was applied and sections were incubated at room temperature for one hour followed by several washings in TBS. Sections were then probed with the primary ALCAM Antibody (1:400 dilution; Novocastra Labs, Newcastle upon Tyne, UK) and then incubated overnight at $4{ }^{\circ} \mathrm{C}$. After several washings, slides were probed with the secondary antibody (Vectastain Elite ABC mouse Kit, Vector Labs, Burlingham, CA, USA) for 30 minutes, washed several times and incubated with the ABCreagent (Vectastatin Elite $\mathrm{ABC}$ mouse Kit) for further 30 minutes and then washed three times. Finally DAB substrate (DAB-Vector-Kit, Vector Labs, Burlingham, CA, USA) was applied for 5 minutes followed by washings in Aqua dest. 
The slides were briefly counterstained with haematoxylin and dehydrated before mounting.

\subsection{Evaluation of the immunohistochemical staining}

To lessen the evaluation bias the histopathological samples has been made anonymous and has been not assigned till the statistical analysis was undertaken. All samples have been IHC stained in two cycles during one week, sorted by organs. The staining was evaluated independently two times by one gynaecologist and one pathologist using the immunoreactive score (IRS) as product of staining intensity (graded between 0 and 3 ) and percentage of positive cells (graded between 0 and 4, nil, 1: < 20\%, 2:20<50\%; 3: 50-80\%, 4: > 80\%) resulting in a score from $0-12$. Evaluation results were summed up and divided by the number of evaluation procedures to receive an average.

\subsection{Statistical analysis}

Mean and standard deviation of ALCAM-score values at each of the sites were calculated. We investigated the intra-class correlation (ICC) to analyse consistency of ALCAM-scores measured at the different locations of metastases within the same patient. Since this value was considerably high, indicating that a large proportion of the variance is explained by the patient identity, we applied a hierarchical regression model where ALCAM expression levels in the metastatic site are predicted based on ALCAM expression levels in the primary tumor, and these values are nested under the patient.

Analyses were calculated using SPSS 14.0 and 16.0 and the $\mathrm{R}$ software. Probability ( $\mathrm{p}$ ) values of $<0.05$ were considered significant.

\section{Results}

\subsection{ALCAM immunostaining in primary and metastases}

\section{Primary tumor}

In normal human breast tissue ALCAM expression shows a patchy membranous and cytoplasmic distribution and variable staining intensity within the terminal duct lobular (TDLU) unit and major ducts using IHC. While the majority of the luminal cells were immunohistochemically positive stained for ALCAM, the most basal cells were negative or only slightly positive. In all
25 but 1 cases of poorly differentiated invasive breast carcinoma we saw ALCAM staining in the tumor. The distribution of the ALCAM expression within the tumor and its staining intensity presented variable and heterogeneous. In two cases we observed a more intense staining at the invasion front of the tumor than in the tumor centre, however without equivalent in the metastases. Exemplary ALCAM staining pattern in two representative cases are shown, see Fig. 2.

\section{Metastases}

Because of the heterogeneous expression of AL$\mathrm{CAM}$ in the primary, we analyzed beside the primary the distant metastases within one single patient to explore whether the expression pattern and staining intensity of ALCAM is similar and whether in dependency of ALCAM expression in the primary there is a preferred site to metastasize detectable.

In all but two cases, which showed no ALCAM staining at all, the tumor cells in the metastases were heterogeneously positive, showing a similar staining intensity compared to the primary site in the same patient. Regarding the staining pattern there were no obvious similarities between primary and metastasis visible. This was due the heterogeneity of size and distribution pattern within the metastatic cell clusters. The evaluation of ALCAM staining in bone metastases was hindered due foregoing decalcification procedures. In these cases the attribution of ALCAM staining to cytoplasm or membrane was indistinct, see Fig. 2.

\subsection{Statistical analysis}

Of the metastatic ALCAM expression levels we obtained an ICC of $80.9 \%$, indicating a strong cluster effect of measurements in the same patient.

When comparing ALCAM expression scores in metastatic sites and in the primary tumor by hierarchical regression analysis we could show that ALCAM expression levels in the primary tumor is prognostic for the average ALCAM expression levels of a patient in all the different sites of metastases (slope $=0.773, p<$ $\left.0.001, r^{2}=0.504\right)$. According to this model, the differences between mean patient's metastasis ALCAM expressions can be explained in $58.1 \%$ by the differences in ALCAM expression in the primary tumor, see Fig. 3. Despite the good explanation there remained an ICC of $63.8 \%$ in the model indicating that there will be further individual patient characteristics that influence the ALCAM expression levels of the metastases. 


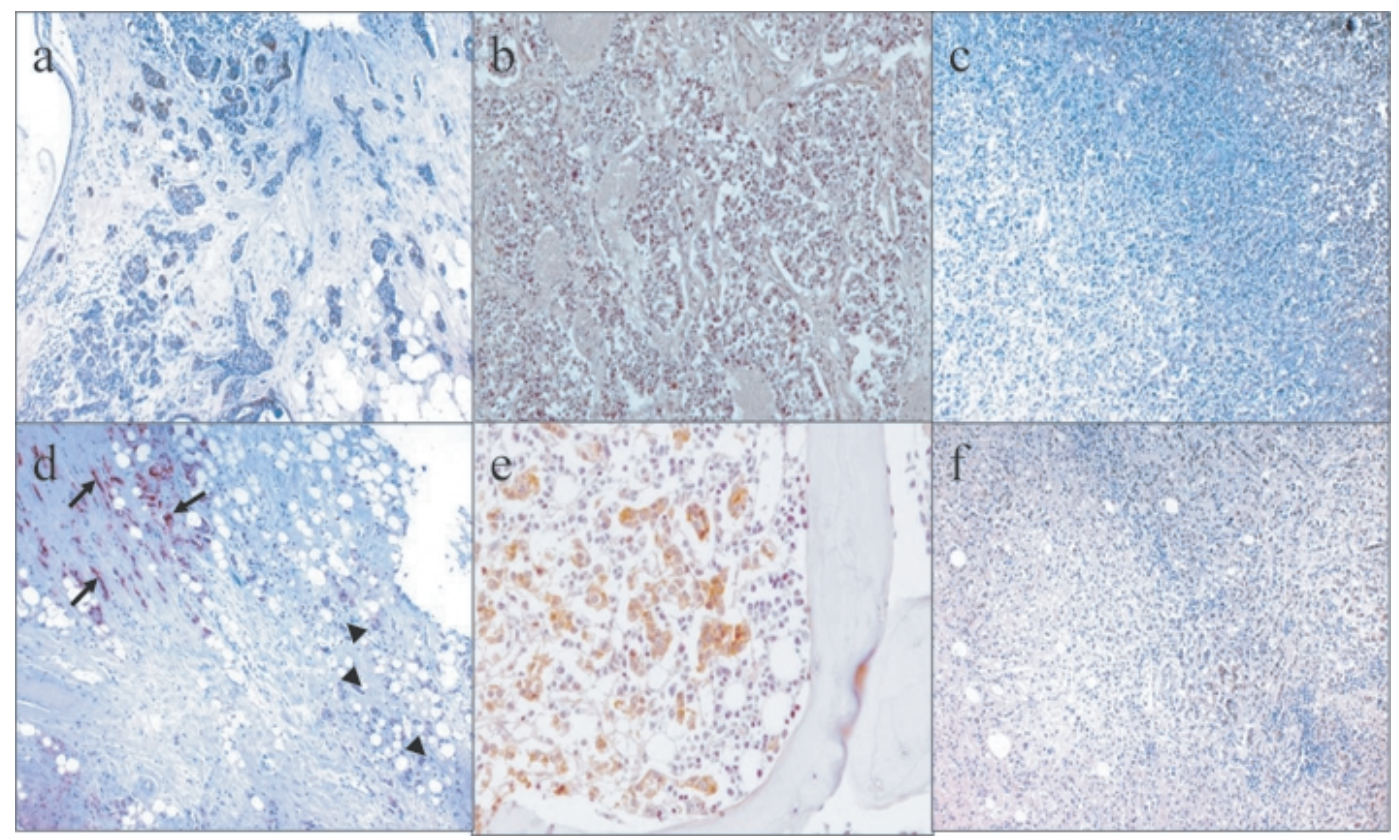

Fig. 2. Immunohistochemical staining for ALCAM in primary invasive breast carcinoma and in its visceral metastasis. Case \#1 (a-c): At primary site the breast carcinoma of invasive ductal type shows an heterogenous expression for ALCAM with intermixed positive and negative tumour cells (a). Beside a weak to moderate cytoplasmic localisation, there is a strong homogenous membranous staining for ALCAM. Taken from the same patient, tumor cells from the metastasis of the lung (b) were throughout negative for ALCAM as well the tumor cells in the liver (c). Normal liver tissue shows a moderate cytoplasmatic staining (upper right). Case \#2 (d-f): Likewise a heterogenous expression for ALCAM is seen in an other primary breast carcinoma of invasive ductal type (d) but here the tumor cells are clearly separated in different areas of the tumor regarding their ALCAM expression. Strong membranous staining in almost all tumor cells is visible in one portion of the tumor (arrows) whereas another portion of the tumor is negative for ALCAM (arrowheads). The tumor cells in metastasis from this cancer in bone (e) and in adrenal gland (f) show a clear membranous ALCAM expression.

The average ALCAM expression levels in different organ sites like mamma (primary) $(\mathrm{N}=25)$, lymph node $(\mathrm{N}=15)$, lung $(\mathrm{N}=19)$, bone $(\mathrm{N}=20)$, liver $(\mathrm{N}=16)$ and adrenal $(\mathrm{N}=7)$ are presented in Fig. 1. Analyses of correlations between the ALCAM expression scores in the primary tumor with patient age and number of metastases or site of metastases did not provide any significant results. There might be no dependency of ALCAM expression in the primary and the preferred site to metastasize detectable, because of the advanced stage of disease, and thus ubiquitous tumor spread.

\section{Discussion}

Adhesion molecules play an important role in interactions of malignant and benign cells. They are supposed to be involved in tumor growth and metastases and therefore important for prognosis and course of disease and may some day be the target for new treatment strategies [2]. ALCAM expression in distant breast cancer metastases or the role of ALCAM levels in the primary tumor regarding to the underlying process of metastases has not been examined yet. All studies concerning ALCAM expression in breast cancer deal with its relevance for prognosis and prediction based on ALCAM expression in the primary tumor. King et al. showed that patients with low levels of ALCAM transcripts had more incidents like metastases, recurrence or death of disease [3] and Jezierska et al. also reasoned that low ALCAM expression levels correlate with aggressive breast cancer and worse outcome assuming that malignant cells could dissolve out of the primary tumor more easily and spread because of the absence of ALCAM as connecting adhesion molecule [6].

In order to analyse a similar cancer profile, we only included poorly differentiated invasive ductal cancers (grade 3 according to Elston and Ellis [22]) into our study. King et al. presumed that ALCAM expression might be lower in higher graded tumors. As King et al. analysed ALCAM on gene level using PCR we could not compare the expression levels precisely. In our study, all primaries are not well differentiated. 


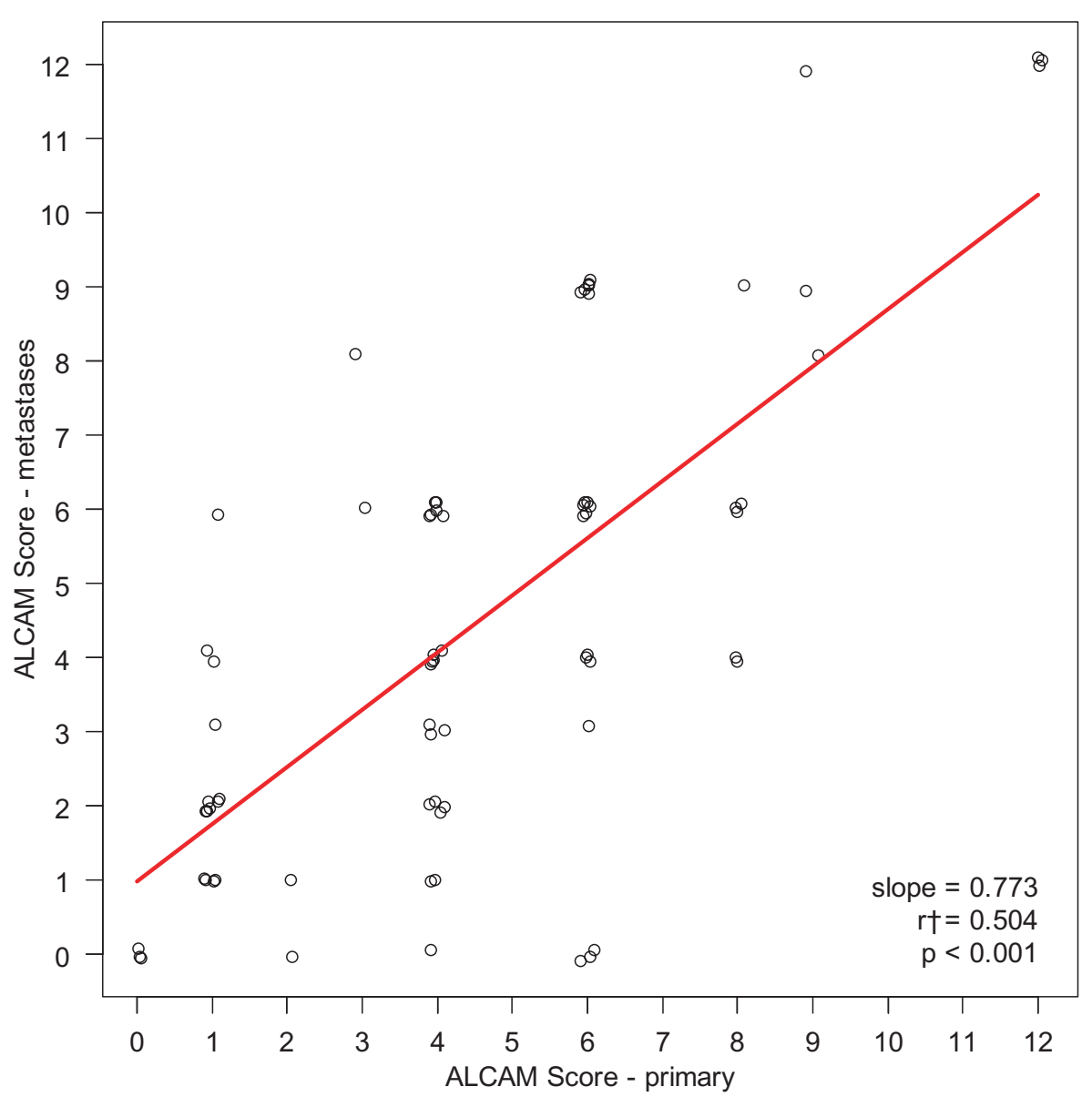

Fig. 3. Regression analysis of ALCAM expression levels in the metastases $(N=84)$ and ALCAM expression levels in the primary (mamma tissue) ( $N=25)$, showing, that differences between mean patient's metastasis ALCAM expressions can be explained in $58.1 \%$ by the differences in ALCAM expression in the primary tumor. Of the metastatic ALCAM expression levels we obtained an ICC of 80.9\%, indicating a strong cluster effect of measurements in the same patient.

We found that the ALCAM expression was normally variable, with an arithmetic mean of 4.32 (0-12) (IRS) within our cohort, which is relatively low. Only one tumor and its metastasis reached an IRS score of 12 and a score of 9 , see Table 1 . This could be a sign that less differentiated tumor cells might have less adhesion abilities. However this has to be further analyzed in different cohorts of patients.

In contrast, Burkhardt et al. found high ALCAM expression in the primary to be a marker for worse prognosis and he additionally suggests that a strong cytoplasmic ALCAM expression, may be the result of an aberrant ALCAM protein expression, because ALCAM normally was supposed to be membranously expressed [17]. Because we analyzed autopsy material, which generally was in good histological condition but showed slight signs of cytolysis, we did not distin- guish between cytoplasmic and membranous staining intensity.

Regarding its predictive value in chemotherapy treated breast cancer patients, Ihnen et al. assigned high ALCAM expression in the primary to be a marker of better therapy response [18]. Apart from data, which deal with prognosis and prediction, only one additional study is available which supposes that patients who develop skeletal metastasis tend to have the lowest levels of ALCAM transcripts in their primary [19]. One of our interests in this study was to evaluate whether there might be a dependency between ALCAM expression level and metastatic site. But as the patients finally died from the disease and the tumor spread within the whole organism, we probably did not see any relation between ALCAM expression and metastatic site anymore. This question should be reviewed in an earlier stage of disease. 
Only very few articles are available dealing with ALCAM and metastases, none of them with breast cancer metastases. Van Kempen et al. analysed melanoma and melanoma metastases and could show ALCAM positivity in half of the examined metastatic tissue [20]. All melanoma metastases were located either in lymph nodes or as skin metastases. No visceral metastases were analysed. The primary melanoma lesion was only available for three single metastases localized in lymph nodes. Both, the primary and the metastases showed weak or no ALCAM expression [20]. In our study, we also found weak or no ALCAM staining in metastases when the primary tumor did not stain for ALCAM or stained weakly, however this was only observed in one case. Altogether the melanoma metastases showed a very weak staining or no staining at all, which differs from our observations on breast cancer samples because our analysed visceral metastasis and lymph node metastases mostly stained very well for ALCAM (see Table 1). Van Kempen et al. stated the hypothesis that down-regulated ALCAM expression in the melanoma metastases might be the result of tumorbiological interactions after the malignant cell clusters have gained access to the metastatic routes [20]. Our data indicate that at least in mammary carcinomas there is no ALCAM down-regulation in distant metastases. In our cohort we showed that the malignant cell clusters expressed high ALCAM levels even beyond the point of spreading.

Other authors detected elevated ALCAM levels in distant metastases of colorectal and appendiceal primaries, however in this small study, they analysed fresh frozen liver and peritoneal metastases tissue using oligonucleotid microarrays [21]. Unfortunately there was no comparison regarding ALCAM expression levels between the gene-profiles of the primary tumor and the metastases performed [21].

In another study it was presumed that ALCAM may play a role in the homing of metastatic disease to i.e. the lung [10]. This was stated because ALCAM was detected in cell cultures of pulmonary microvascular endothelial cells as well as in breast cancer tissue and is known to be involved in homophilic adhesion which may result in the adhesion of a single breast cancer cell circulating in the blood stream to the pulmonary vessels and lead to invasive growth and metastases [10]. Based on this, one could suppose that the local ALCAM expression in a single organ might determine a favored pattern of metastases sites but as mentioned above this should be revealed in another study design.

ALCAM is expressed in a great variety of adult and embryotic human and animal tissues. It was detected in brain, bone marrow, lymphoid cells, lung and hepatocytes [5]. All of these organs are known to be predestined for breast cancer metastases nesting. Unfortunately in the published articles ALCAM expression has been described, but there is no model available, which allows to infer, if organ- or tumorcells expressing high amounts of ALCAM might be predestined for an increased homophilic ALCAM binding and favoured cell adherence. In summary less is known regarding adhesion molecules and its function concerning the development of metastatic disease in breast cancer, but there are indications that adhesion molecules like ALCAM may play an important role in this process. This leads to the consideration that antibody therapies might be a worthwhile therapeutic approach for patients suffering from progressive disease.

\section{References}

[1] W. Degen, L. van Kempen, E.G. Gijzen, J.J. van Groningen, Y. van Kooyk, H.P. Bloemers and G.W. Swart, MEMD, a new cell adhesion molecule in metastasizing human meanoma cell lines is identical to ALCAM (activated leukocyte cell adhesion molecule), Am J Pathol 152 (1998), 805-813.

[2] W. Jiang, M. Puntis and M.B. Hallett, Molecular and cellular basis of cancer invasion and metastasis: implications for treatment, Br J Surg 81 (1994), 1576-1590.

[3] J. King, S. Ofori-Acquah, T. Stevens, A. Al-Mehdi, O. Fodstad and W.G. Jiang, Activated Leukocyte Cell Adhesion Molecule in breast cancer: prognostic indicator, Breast Cancer Res 6 (2004), 478-487.

[4] L. van Kempen, J. Nelissen, W.G.J. Degen, R. Torensma, U.H. Weidle, H.P.J. Bloemers, C.G. Figdor and G.W.M. Swart, Molecular Basis fo the homophilic Activated Leukocyte Cell Adhesion Molecule (ALCAM)-ALCAM interaction, The Journal of Biological Chemistry 276 (2001), 2578325790.

[5] G. Swart, Activated Leukocyte Cell Adhesion Molecule (CD166/ALCAM): Developmental and mechanistic aspects of cell clustering ans cell migration, European Journal of Cell Biology 81 (2002), 313-321.

[6] A. Jezierska, P. Wojciech, J. Pietruszkiewicz, W. Olszewski, W. Matysiak and T. Motyl, Activated Leukocyte Cell Adhesion Molecule (ALCAM) is associated with suppression of breast cancer cells invasion, Med Sci Monit 12 (2006), 245-256.

[7] S. Bruder, N. Ricalton and R.E. Boynton, Mesenchymal stem cell surface antigen SB-10 corresponds to activated leukocyte cell adhesion molecule and is involved in osteogenic differentiation, J Bone Mineral Res 13 (1998), 655-663.

[8] M. Brown, A. Tsodikov, K.R. Bauer, C.A. Parise and V. Caggio, The role of epidermal growth factor receptor 2 in the survival of women with estrogen and progesterone receptornegative, invasive breast cancer, Cancer 112 (2008), 737-747.

[9] O. Ohneda, K. Ohneda and F. Arai, ALCAM (CD166): its role in hematopoietic and endothelial development, Blood 98 (2001), 2134-2142.

[10] J. King, S. Ofori-Acquah, T. Stevens and A. Al-Mehdi, Potential role for Activated Leukocyte Cell Adhesion Molecule and 
Neural Cadherin in metastasis to the lung microcirculation, Chest 125 (2004), 150S-151S.

[11] P. Lunter, J. van Kilsdonk, H. van Beek, I.M.H.A. Cornelissen, M. Bergers, P.H.G.M. Willems, G.N.P. van Muijen and G.W.M. Swart, Activated Leukocyte Cell Adhesion Molecule (ALCAM/CD166/MEMD), a novel actor in invasive growth, controls Matrix Metalloproteinase activity, Cancer Res $\mathbf{6 5}$ (2005), 8801-8808.

[12] G. Kristiansen, C. Pilarsky, C. Wissmann, C. Stephan, L. Weissbach, V. Loy, S. Loening, M. Dietel and A. Rosenthal, ALCAM/CD166 is up-regulated in low-grade prostate cancer and progressivley lost in high-grade lesions, Prostate $\mathbf{5 4}$ (2003), 34-43.

[13] W. Weichert, T. Knösel, J. Bellach, M. Dietel and G. Kristiansen, ALCAM/CD166 is overexpressed in colorectal carcinoma and correlates with shortened patient survival, Journal of Clinical Pathology 57 (2004), 1160-1164.

[14] A. Verma, N. Shukla, S.V.S. Deo, S.D. Gupta and R. Ralhan, MEMD/ALCAM: A potential marker for tumor invasion and nodal metastasis in esophageal squamous cell carcinoma, Oncology 68 (2005), 462-470.

[15] G. Swart, P. Lunter, J.W.J. van Kilsdonk and L.C.L.T. van Kempen, Activated leukocyte cell adhesion molecule (ALCAM/CD166): signaling at the divide of melanoma cell clustering and cell migration? Cancer Met Rev 24 (2005), 223236.

[16] C. Riley, D. Mahadevan, J.W. Saldanha, W. Qi, L.S. Cooke, Y. Zhu, S.T. Narayan, K. Shakalya, K.D. Croce, I.G. Georgiev, R.B. Nagle, H. Garewal, D.D. von Hoff and D. Mahadevan, Design and activity of a murine and humanized anti-
CEACAM6 single-chain variable fragment in the treatment of pancreatic cancer, Cancer Res 69 (2009), 1933-1940.

[17] M. Burkhardt, E. Mayordomo, K.J. Winzer, F. Fritzsche, T. Gansukh, S. Pahl, W. Weichert, C. Denkert, H. Guski, M. Dietel and G. Kristiansen, Cytoplasmatic overexpression of ALCAM is prognostic of disease progression in breast cancer., J Clin Pathol 59 (2006), 403-409.

[18] M. Ihnen, V. Müller, R.M. Wirtz, C. Schröder, S. Krenkel, I. Witzel, B. Lisboa, F. Jänicke and K. Milde-Langosch, Predictive impact of Activated Leukocyte Cell Adhesion Molecule (ALCAM/CD166) expression in breast cancer, Breast Cancer Res Treatm 112 (2008), 419-427.

[19] S. Davies, C. Dent, G. Watkins, J.A. King, K. Mokbel and W.G. Jiang, Expression of the cell to cell adhesion molecule, ALCAM, in breast cancer patients and the potential link with skeletal metastasis, Oncol Rep 19 (2008), 555-561.

[20] L. van Kempen, J. van den Oord, G.N.P. van Muijen, U.H. Weidle, H.P.J. Bloemers and G.W.M. Swart, Activated leukocyte adehsion molecule/CD166, a marker of tumor progression in primary malignant melanoma of the skin, Am J Pathol 156 (2000), 769-774.

[21] S. Varghese, M. Burness, H. Xu, T. Beresnev, J. Pingpank and H.R. Alexander, Site-specific gene expression profiles and novel molecular prognostic factors in patients with lower gastrointestinal adenocarcinoma diffusely metastatic to liver or peritoneum, Ann Surg Oncol. 14 (2007), 3460-3471.

[22] C.W. Elston and I.O. Ellis, Pathological prognostic factors in breast cancer: The value of histological grade in breast cancer: experience from a large study with long-term followup, Histopathology 19 (1991), 403-410. 


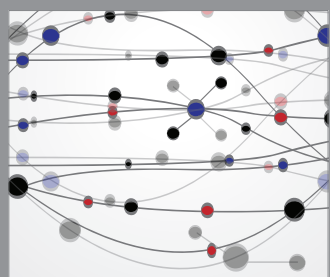

The Scientific World Journal
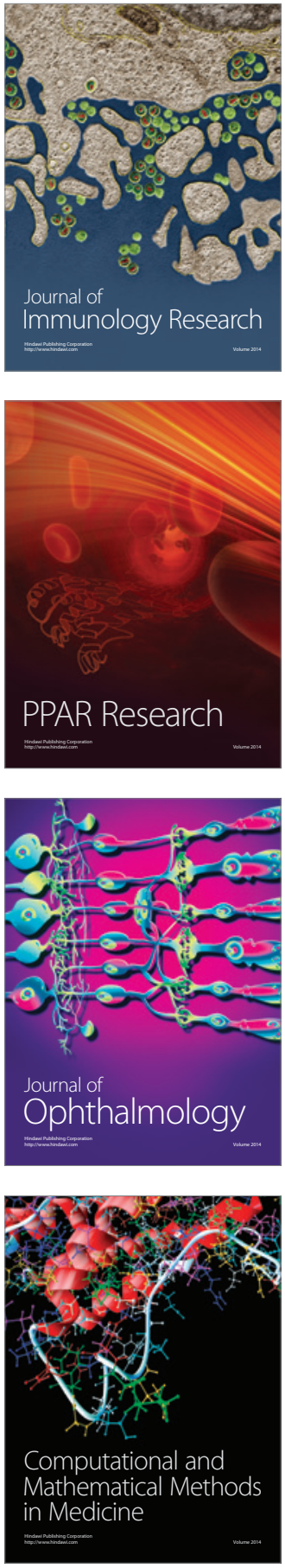

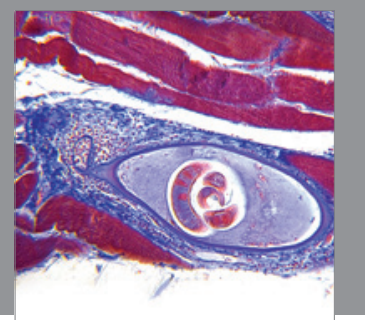

Gastroenterology

Research and Practice
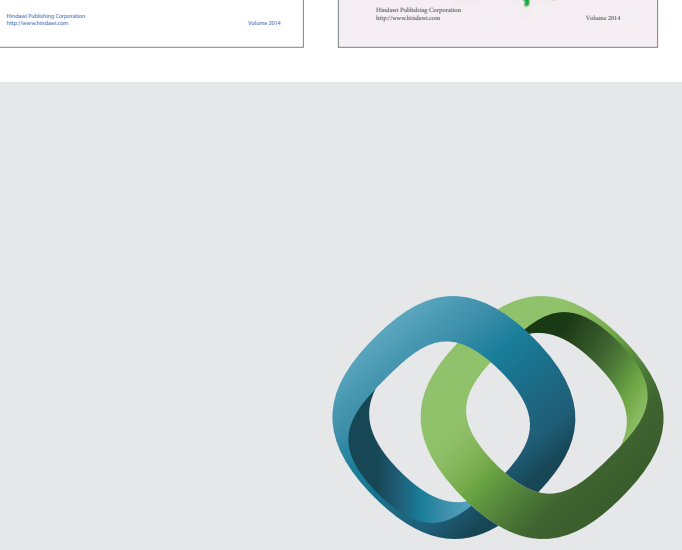

\section{Hindawi}

Submit your manuscripts at

http://www.hindawi.com
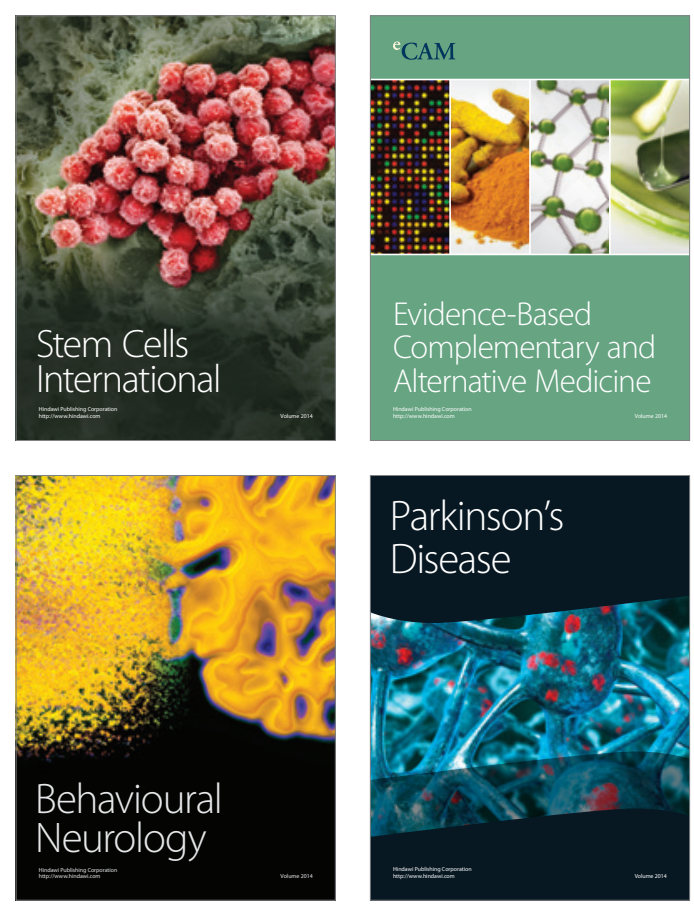

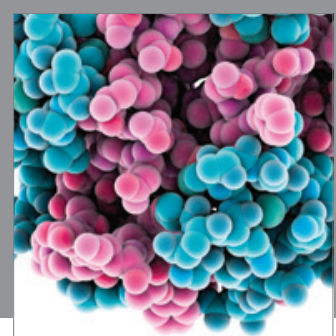

Journal of
Diabetes Research

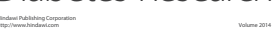

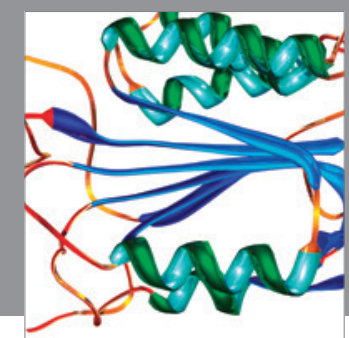

Disease Markers
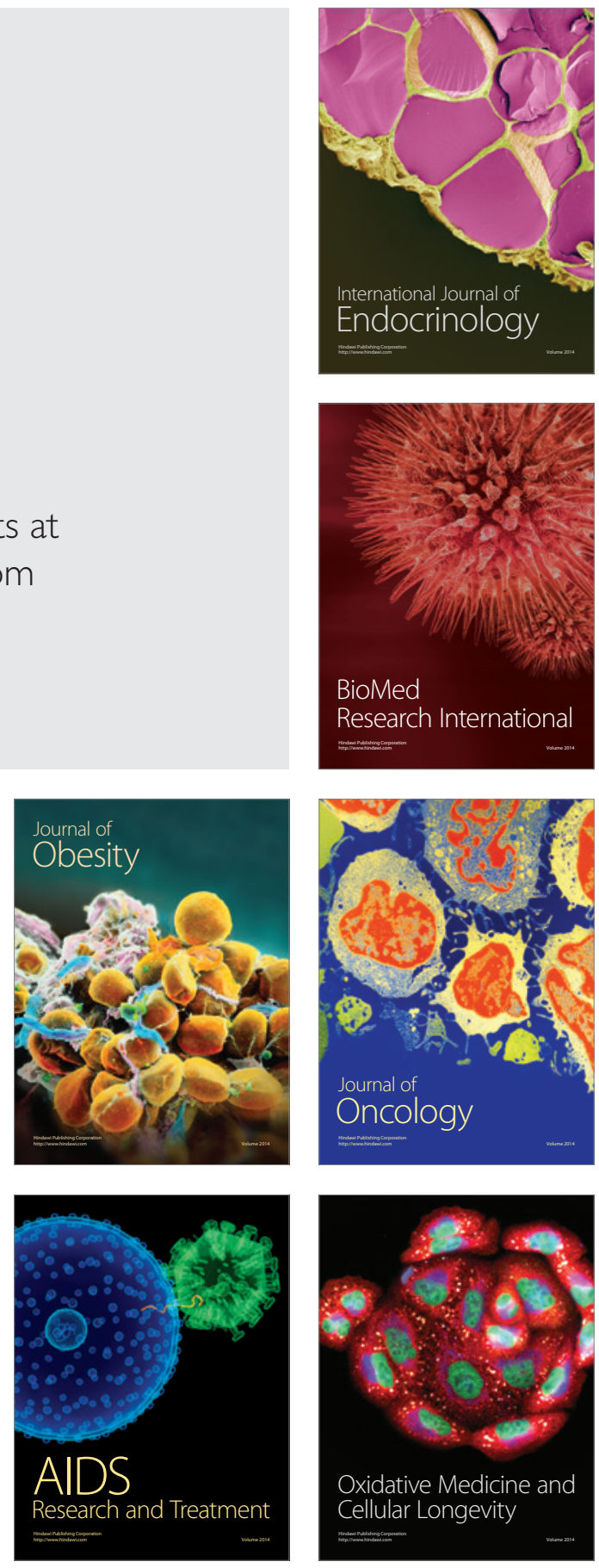